\title{
Network Recasting: A Universal Method for Network Architecture Transformation
}

\author{
Joonsang Yu, Sungbum Kang, Kiyoung Choi \\ Dept. of Electrical and Computer Engineering \\ Neural Processing Research Center (NPRC) \\ Seoul National University, Seoul, Korea \\ \{joonsang.yu, sb05kang\}@dal.snu.ac.kr, kchoi@snu.ac.kr
}

\begin{abstract}
This paper proposes network recasting as a general method for network architecture transformation. The primary goal of this method is to accelerate the inference process through the transformation, but there can be many other practical applications. The method is based on block-wise recasting; it recasts each source block in a pre-trained teacher network to a target block in a student network. For the recasting, a target block is trained such that its output activation approximates that of the source block. Such a block-by-block recasting in a sequential manner transforms the network architecture while preserving the accuracy. This method can be used to transform an arbitrary teacher network type to an arbitrary student network type. It can even generate a mixed-architecture network that consists of two or more types of block. The network recasting can generate a network with fewer parameters and/or activations, which reduce the inference time significantly. Naturally, it can be used for network compression by recasting a trained network into a smaller network of the same type. Our experiments show that it outperforms previous compression approaches in terms of actual speedup on a GPU.
\end{abstract}

\section{Introduction}

Deep Neural Networks (DNNs) are widely used for many kinds of recognition and classification tasks because it has outperformed previous machine learning methods in terms of inference accuracy. New kinds of DNN architecture have been introduced to achieve even higher accuracy (Lin, Chen, and Yan 2014; Szegedy et al. 2015; Larsson, Maire, and Shakhnarovich 2017; He et al. 2016; Zagoruyko and Komodakis 2016; Huang et al. 2017), and the networks become deeper and deeper to take the exponential advantage of depth (Goodfellow et al. 2016). To train a deep network, He et al. (2016) proposed the residual network (ResNet), which consists of the summation of identity mapping and output of convolutional layers. It helps to propagate gradients from top layer to bottom layer, so it can alleviate the vanishing-gradient problem. The densely connected network (DenseNet) is also proposed to solve that problem and improve information flow (Huang et al. 2017). DenseNet uses the feature concatenation method instead of summation, so bottom layers can access gradients directly through the concatenation path.

Copyright (c) 2019, Association for the Advancement of Artificial Intelligence (www.aaai.org). All rights reserved.
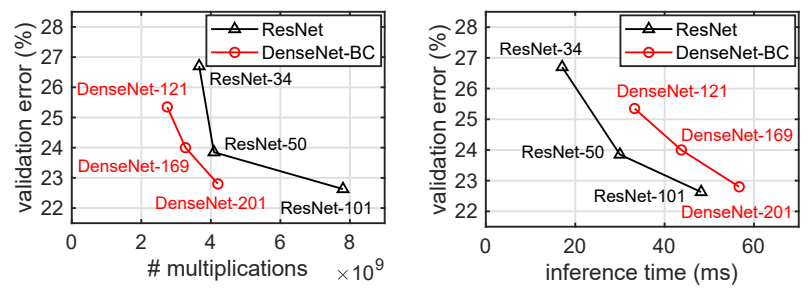

Figure 1: ResNet and DenseNet Top-1 validation errors for different numbers of multiplications (left) and inference times (right). To measure the inference time, single NVIDIA Titan X (Pascal) is used and batch size is set to 16. DenseNet has much fewer multiplications than ResNet, but its inference time is much longer.

Deeper network architectures help to achieve higher accuracy, but those have a huge amount of parameters and computation redundancies. To design a compact network architecture, the $1 \times 1$ convolution is added (Szegedy et al. 2015; He et al. 2016; Huang et al. 2017). The additional $1 \times 1$ convolution reduces the number of channels of output activation. The number of parameters and multiplications in the $3 \times 3$ convolution are also reduced thanks to the $1 \times 1$ convolution. In this reason, bottleneck block in ResNet and dense block in DenseNet use the $1 \times 1$ convolution for the parameter and multiplication reduction.

However, the bottleneck and dense block actually increase inference time even though the number of multiplications is reduced. Figure 1 shows the number of multiplications and actual inference time for three models of ResNet and DenseNet. ResNet-50 has a number of multiplications similar to that of ResNet-34 thanks to the $1 \times 1$ convolution, but it takes $1.8 \times$ longer than ResNet-34. This is because the bottleneck block of ResNet has four times larger activation map compared with basic residual block, so it causes four times more activation load from off-chip memory. Although DenseNet has a much smaller number of parameters and multiplications compared with ResNet, its inference time is much longer than that of ResNet. DenseNet has much smaller total activations than ResNet, but actual activation load of DenseNet is much larger than that of ResNet because the layers in DenseNet use output activations of all previous 
layers and thus actual activation load is much larger than the total activation size.

In this paper, we focus on the inference time reduction rather than parameter and multiplication reduction. To reduce the inference time, we propose the network recasting method by transforming the network architecture for smaller activation load. We transform the network architecture through block-wise recasting of source blocks into target blocks. The recasting is done by training the target block to mimic the output activation of the source block, so the accuracy can be preserved after recasting. We can obtain a mixed-architecture network by recasting parts of the trained network. By the mixed-architecture network, we mean a network having multiple types of block that can exploit the advantages of individual block types within a single network. In addition, we can use the network recasting method for network compression by recasting each block to a smaller one of the same type. We have achieved up to $3.2 \times$ actual speedup with $0.22 \%$ top-5 accuracy loss on ILSVRC2012 dataset by the DenseNet-121 recasting.

\section{Related Works}

Network pruning To reduce the size and inference time of a trained network, several pruning methods such as weight pruning and filter pruning have been proposed. Han et al. (2015) propose an iterative weight pruning method that removes connections and neurons according to the absolute value of parameters. Guo, Yao, and Chen (2016) also propose iterative weight pruning that also gives a chance to restore connections for pruned weight. However, weight pruning methods generate sparse parameter matrices rather than smaller matrices, so its actual speedup is much less than the parameter reduction in general purpose hardware (Liu et al. 2015). The filter pruning methods reduce the size of parameter and activation matrices after the pruning, so they are more effective to accelerate the inference in any kinds of hardware. To find filters to be pruned, average percentage of zeros (APoZ), sum of absolute values, and reconstruction error of activation are used (Hu et al. 2016; Li et al. 2016; Luo, Wu, and Lin 2017; He, Zhang, and Sun 2017). Luo, $\mathrm{Wu}$, and Lin (2017) find and remove filters that have the smallest influence on the output activation of the next layer, and He, Zhang, and Sun (2017) train a channel pruning mask minimizing the reconstruction error of current output activation and prune the channel of filters according to the trained mask. Liu et al. (2017) and Luo and Wu (2018) use channel scaling factor by adapting additional trainable parameters or squeeze-and-excitation layer (Hu, Shen, and Sun 2018), and then prune filters according to the scaling factor. Recently, Lin et al. (2017) use deep reinforcement learning to select pruning candidates at runtime.

Knowledge distillation To train a smaller network with higher accuracy, mimic learning and knowledge distillation (KD) are introduced by $\mathrm{Ba}$ and Caruana (2014) and Hinton, Vinyals, and Dean (2014), respectively. These methods train a smaller network called student network using logits of a large network called teacher network. Ba and
Caruana (2014) train a student network by minimizing L2 loss between logits of student and teacher networks. Hinton, Vinyals, and Dean (2014) use logits of the teacher network to generate soft target, and train student network by minimizing cross-entropy loss with the soft target. It is hard to train a deep student network due to the vanishinggradient problem, so several KD methods have been proposed to train a deep student network (Romero et al. 2015; Luo et al. 2016). To train a thinner and deeper student network using KD, Romero et al. (2015) propose hint training that trains a hidden layer with a convolutional regressor. Luo et al. (2016) make additional paths from a hidden layer to the output layer for gradient propagation without vanishing. In addition, Zagoruyko and Komodakis (2017) introduce the attention transfer method to reduce the number of residual blocks while conserving the accuracy. Yim et al. (2017) also propose the residual block reduction method using the relationship between input and output activations. Also, there is a recent research to train the ResNet using logits of DenseNet (Furlanello et al. 2018).

Key differences Our work is for general recasting of neural networks. It can be used in various ways such as network size reduction or network type transformation. Compared to the previous work on network size reduction using weight/filter pruning, our work is different in that the inference process of the reduced network can be made significantly faster through the reduction of activations. We also reveal the fact that reducing activation size is more important for inference speed than reducing the number of parameters. Compared to other approaches using the knowledge distillation technique, our work is different in that the technique is applied sequentially to further enhance the accuracy.

\section{Network Recasting}

The network recasting method recasts a pre-trained network into a network of different type and/or size. Given the pretrained teacher network, we transform each block (source block) in the teacher network into a new block (target block) of pre-defined type and size in the student network. The transformation is done by training the target block to generate output activations similar to those of the source block. We call this process block recasting. In this process, the source block can be considered as an unknown function, and the target block can be considered as a functional approximator similar to a multilayer perceptron (Hornik 1991). After recasting all candidate blocks, we obtain the student network, which is faster than the teacher network while preserving the functionality or accuracy. We call the entire process network recasting.

\section{Recasting from DenseNet to ResNet and ConvNet}

The DenseNet has a lot of activation load due to the dense connection, and by recasting the dense block into a basic residual block (we call the basic residual block as basic block for simplicity), we can reduce the inference time. We consider a basic block consisting of two $3 \times 3$ convolution 


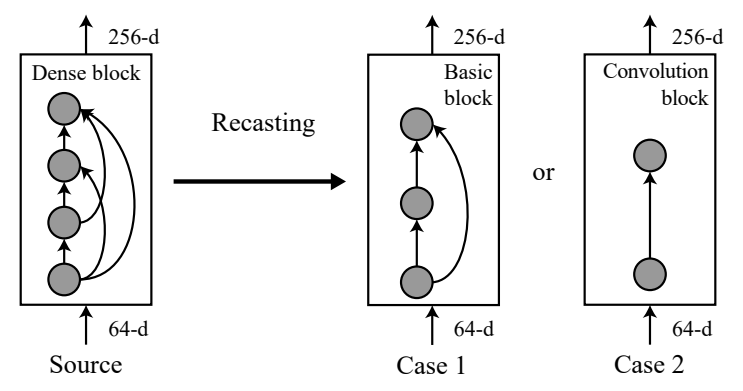

Figure 2: Block recasting of a dense block into a basic block (Case 1) and a convolution block (Case 2). The basic block has shorter inference time than the dense block because it has much smaller activation load. The convolution block is even faster than the basic block, but its capacity is much smaller and so it can cause accuracy loss.
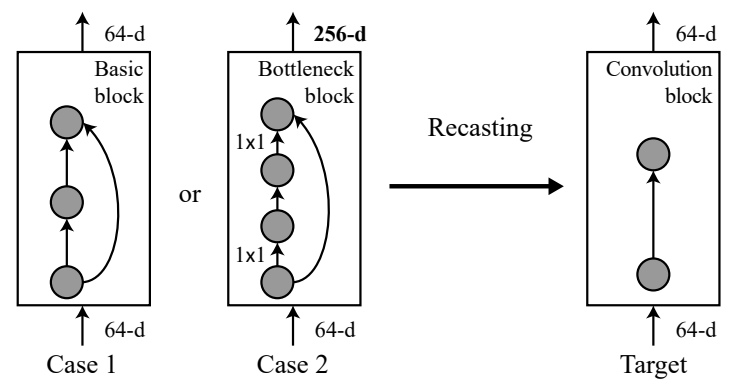

Figure 3: Block recasting of a residual block-basic block (Case 1) and bottleneck (Case 2) — into a convolution block. The recasting of the basic block keeps the same number of input and output channels. However, since the bottleneck block uses a smaller number of channels for the feature extraction, we recast it into a convolution block that has the same number of input and output channels as the original $3 \times 3$ convolution.

and shortcut as shown in Figure 2. Even though the basic block has more parameters and multiplications than the dense block, its activation load is much smaller and thus it is much faster. For more inference time reduction, we can recast the dense block into a single convolution block, although it can cause more accuracy loss because it has a very small capacity. Figure 2 shows the two examples of recasting the first dense block in DenseNet-121.

\section{Recasting from ResNet to ConvNet}

Figure 3 illustrates the block recasting of a residual block into a convolution block. In the basic block, local features are extracted from the input activations using $3 \times 3$ filters, and thus, we recast the basic block into a $3 \times 3$ convolution block. Since the new convolution block has the same number of filters as the original basic block, the dimension of the output activations is not changed. However, in bottleneck block recasting, the dimension of the output activation is reduced as shown in Figure 3 (Case 2) for the first bottleneck block of ResNet-50. Although the output activation becomes smaller, the number of linearly independent fea-
Table 1: Candidates for the network recasting.

\begin{tabular}{lccc}
\hline Recasting Type & Source & Target & Dimension \\
\hline \multirow{4}{*}{ Transformation } & Dense & Basic & Preserved \\
& Dense & Convolution & Preserved \\
& Basic & Convolution & Preserved \\
& Bottleneck & Convolution & Reduced \\
\hline \multirow{2}{*}{ Compression } & Basic & Basic & Reduced \\
& Convolution & Convolution & Reduced \\
\hline
\end{tabular}

tures is not changed because the second $1 \times 1$ convolution in the source block just combines its input activations linearly to extend the dimension of output activation. Therefore, the next block in the student network still can reconstruct similar activation map.

\section{Compression}

The network recasting can be used to compress the large network while preserving accuracy. In this case, we assume that the network has redundancy such as ineffectual filters and redundant filters. An ineffectual filter denotes a filter that cannot extract any meaningful feature, and a redundant filter denotes a filter that extracts a feature very similar to the one extracted by some other filter or a feature that can be obtained by combining features from other filters. To remove those filters, previous approaches use APoZ (Hu et al. 2016), sum of absolute values of a filter ( $\mathrm{Li}$ et al. 2016), or influence on next activations (Luo, Wu, and Lin 2017; He, Zhang, and Sun 2017) as the criteria, but redundant filters cannot be founded with those approaches. A possible approach is to find such redundant filters by checking the similarity between every pair of filters. However, it requires a huge amount of computations for similarity check and does not guarantee a good result. Instead, we recast a given source block into a smaller target block that has the same type as the source block. Then we train the target block and the next block to reconstruct the output activation of the next block with smaller number of filters. If the next block can reconstruct a similar output activation, the new target block can extract effective features for reconstruction. For example, a convolution block is recast into another convolution block that has a smaller number of filters. Then we train both the new convolution block and the next block to reconstruct the orginal activation map of the source next block. After training, we can obtain a more effective filter set without any similarity or effectiveness check criteria.

\section{Block Training}

For the target block to work properly, it should be trained with the source block as the teacher. We can easily train the target block by approximating the output activations to those of the source block if both blocks have the same dimension of output activations. However, dimension mismatch happens in the block recasting especially when we reduce the number of channels for network size reduction. Table 1 shows the recasting cases that we handle in this paper. To avoid the dimension mismatch problem, when training a target block, we train the target block together with the next 


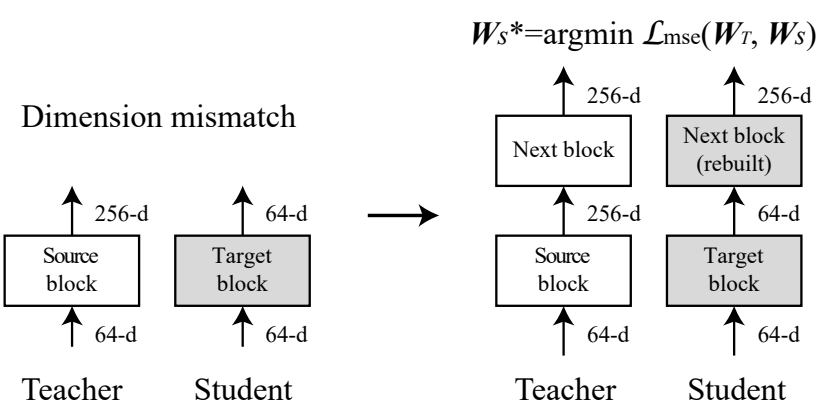

Figure 4: The dimension mismatch happens when the source block is recast into a smaller target block. The next block is used to match the dimension of output activation. After rebuilding the next block, both blocks are trained by minimizing $\mathcal{L}_{m s e}\left(W_{T}, W_{S}\right)$.

block by approximating the output activations of the next block as shown in Figure 4. The next block is rebuilt from the corresponding source block by reducing the filter size when the target block has a smaller number of channels. Both the target block and the next block are initialized randomly and trained to minimize the loss of mean-square error (MSE) between teacher's and student's activations given by,

$$
\mathcal{L}_{m s e}\left(W_{T}, W_{S}\right)=\frac{1}{N}\left\|A\left(x ; W_{T}\right)-A\left(x ; W_{S}\right)\right\|_{2}^{2},
$$

where $A$ means the activation of the next block, and $x$ is the input data. $W_{T}$ and $W_{S}$ indicate parameters of teacher network and student network, respectively. $N$ denotes the size of an output activation of the next block.

\section{Sequential Recasting and Fine-tuning}

To recast the entire network, we apply the block recasting method sequentially. Figure 5 shows an example of sequential recasting method. The type and dimension of the first (target) block of the student network are determined, and then the second block is rebuilt from the second block of the teacher network; if there is no dimension mismatch, the second block will be the same as that of the teacher network. The two blocks are initialized randomly and trained by minimizing $\mathcal{L}_{m s e}\left(W_{T}, W_{S}\right)$. Now, the second block becomes the target. Thus, its type and dimension are determined, the third block is rebuilt, and both blocks are initialized randomly. To train the second and third blocks, we reuse the trained first block. The first block is already trained in the previous step, but it still has approximation errors. We can reduce the effect of its errors by training both the previous and current blocks. Therefore, three blocks are trained in the second step by minimizing $\mathcal{L}_{m s e}\left(W_{T}, W_{S}\right)$. This process is continued for the following blocks until the last block is recast as a new block. We can select arbitrary blocks as candidates for recasting so that the student network can consist of multiple types of block. For example, the student network can have both residual and dense blocks when only the first dense block is recast into a residual block. We call the network that has multiple types of block as mixed-architecture network. The mixed-architecture network can have advantages of both blocks. For example, by mixing dense blocks and residual blocks, we can obtain a mixed-architecture network that is faster than DenseNet and has fewer parameters than ResNet.

The block-by-block sequential recasting has two advantages. First, the functionality of each block is much simpler than that of the whole network. Thus, it is easier to approximate the functionality of each block. By approximating each of easier sub-functions, we can finally obtain the student network with smaller approximation error. Secondly, sequential recasting can alleviate the vanishing-gradient problem. When the source block is recast as a convolution block, the student network cannot be trained well due to the gradient vanishing. However, sequential recasting has very short gradient paths from the output activation to the target block, so it can be trained well. Therefore, we can obtain the student network with higher accuracy using sequential recasting.

After finishing sequential recasting, we use the knowledge distillation approach to fine-tune the student network. There are approximation errors after sequential recasting, and we can reduce the effect of those errors by training the whole network. We train the student network with logits of the teacher network and ground truth. Thus, our knowledge distillation (KD) loss is defined by

$$
\mathcal{L}_{k d}\left(W_{T}, W_{S}\right)=\mathcal{L}_{\text {mse_logit }}\left(W_{T}, W_{S}\right)+\mathcal{L}_{c e}\left(y_{\text {true }}, W_{S}\right),
$$

where $\mathcal{L}_{\text {mse_logit }}$ is the MSE loss for the logits, and $\mathcal{L}_{c e}$ is the cross-entropy loss between the given label $y_{\text {true }}$ and softmax output of the student network that is parameterized by $W_{s}$.

\section{Experiments}

We conducted several experiments for the network recasting. For the experiments, we used CIFAR and ILSVRC2012 dataset and four kinds of network architectures; ResNet (He et al. 2016), Wide ResNet (WRN) (Zagoruyko and Komodakis 2016), DenseNet (Huang et al. 2017), and VGG16 (Simonyan and Zisserman 2015). We adopted batch normalization (Ioffe and Szegedy 2015) for all networks, because it was also effective for block-wise training. The network recasting was implemented on the PyTorch framework. We used the Xavier initializer (Glorot and Bengio 2010) in all experiments. We used SGD with Nesterov momentum (Sutskever et al. 2013) to train the teacher network and used Adam optimizer (Kingma and Ba 2015) for the network recasting. In addition, we trained the student network with KD and back propagation from scratch using SGD with Nesterov momentum for the comparison.

\section{Visualization of Filter Reduction}

The network recasting can be used for network compression; it can remove redundant filters as well as ineffectual filters. To show the filter reduction, we compressed only the first layer of AlexNet and visualized the filter set in Figure 6. The first layer of the original AlexNet had 64 filters, but we decreased the number to 25 in the student network. Then we trained the first block of the student network 


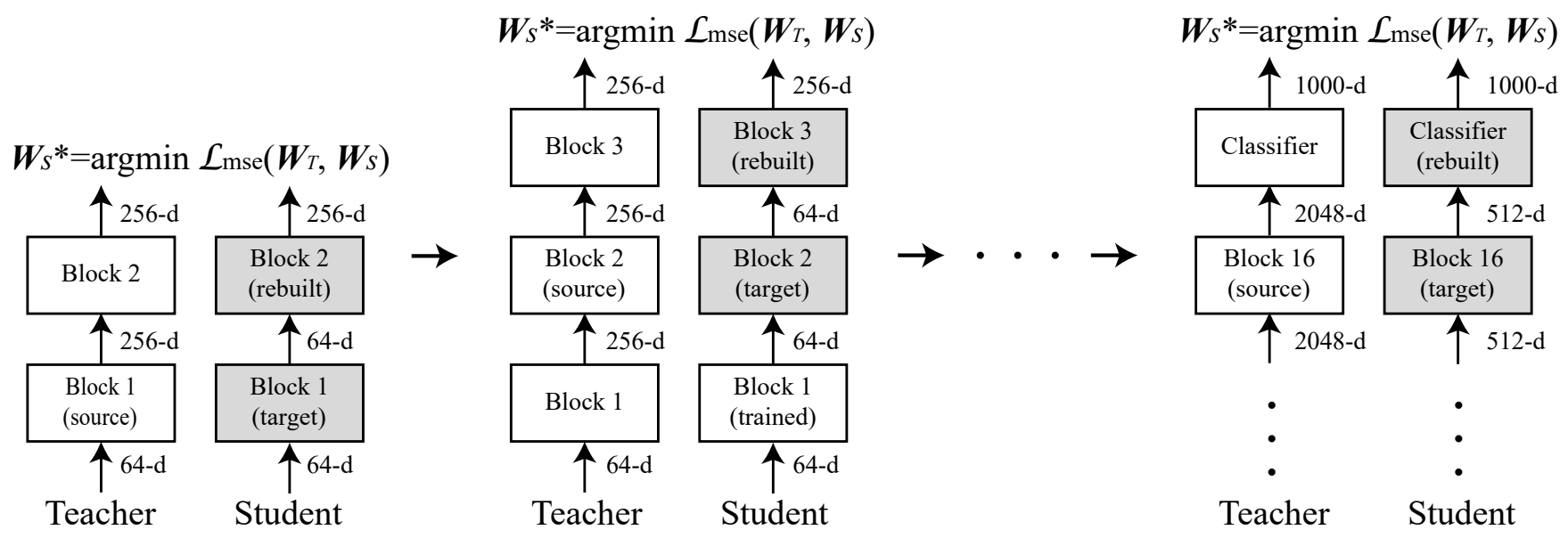

Figure 5: An example of sequential recasting for ResNet-50. All blocks are recast in this example. In each step, the target block and the next block (shaded blocks) are initialized randomly and trained by minimizing $\mathcal{L}_{m s e}\left(W_{T}, W_{S}\right)$.

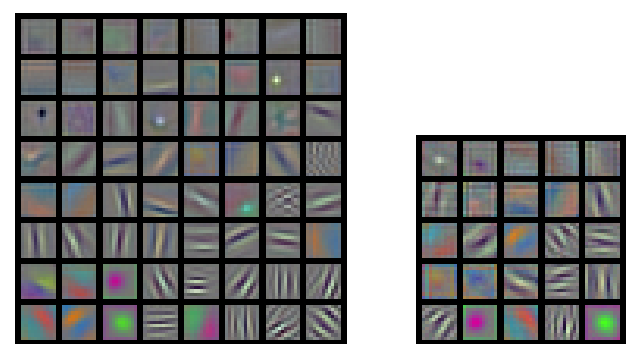

Figure 6: Visualization of filters in the first layer of AlexNet (left) and a student network (right). Redundant filters are removed after network recasting.

for eight epochs, and fine-tuned the entire student network; the learning rates for the recasting of the first block and the fine-tuning were 0.0005 and 0.0001 , respectively. Every five epochs, the running rates were divided by 10 . Figure 6 shows filters extracted from the first layers of the teacher and student networks. Filters of the teacher network consist of many ineffectual and redundant filters, but those are eliminated as shown in Figure 6. In addition, the student network achieves the top- 1 error of $44.20 \%$ and the top-5 error of $21.54 \%$. The top- 1 and the top- 5 errors increase by only $0.72 \%$ and $0.61 \%$, respectively. Note that it is hard to remove many filters without accuracy loss because AlexNet has a relatively large $(11 \times 11)$ filters. The filter size is related to the dimension of filter vector, and many more filters are required to span the vector space as the filter size increases. As expected, we could remove many more filters on both VGG-16 and ResNet, which have only $3 \times 3$ filters.

\section{CIFAR}

For CIFAR dataset, we used ResNet-56, ResNet-83, WRN28-10, DenseNet-100, and VGG-16. Especially, ResNet-83 has the same number of blocks with ResNet-56, but consists of bottleneck blocks. In addition, we used a modified version of VGG16, which has only one hidden fully-connected layer with 512 neurons. Teacher networks were trained from scratch using back propagation. We used CIFAR-10 and 100 dataset with the standard data augmentation, which consists of four pixel zero-padding and random cropping, and horizontal flipping with 0.5 probability.

In CIFAR experiments, we recast all blocks of teacher networks, so there is no mixed-architecture result. We counted the number of parameters, multiplications, and activation loads for the convolution operation. Especially, we reported the activation load of a single image in Table 2. Table 2 shows the architecture transformation results. The network recasting achieved similar accuracy with the teacher network, and activation access is reduced significantly. It shows lower test error compared to other methods in all network architectures. When networks were recast into a plain convolutional network, the network recasting achieved much lower test error compared with both $\mathrm{KD}$ and back propagation. The sequential recasting can alleviate the vanishing-gradient problem, so its results outperformed the others.

We also compressed the VGG-16 and WRN-28-10 using the network recasting. In this experiment, source blocks were recast into $2.5 \times$ and $5 \times$ smaller blocks in VGG-16 and WRN-28-10, respectively. Table 3 shows compression results of both networks. The network recasting achieved the smallest accuracy loss compared with other methods. Especially, network recasting achieved $1.58 \%$ and $3.57 \%$ lower test error compared with KD and back propagation in VGG16 compression on CIFAR-100.

The born again network (BAN) proposed by (Furlanello et al. 2018) also trains ResNet student using logits of DenseNet teacher. However, they proposed only switching DenseNet with ResNet, and the test error of BAN will be higher than that of network recasting because BAN only uses the KD method as shown in Table 2 and 3. We propose any to any architecture transformation, and deep student networks that have only convolution blocks can also be trained well by applying sequential recasting because it can alleviate the vanishing-gradient problem. In addition, we also propose mixed-architecture network, which can also be trained well by using the proposed network recasting. 
Table 2: Error rates (\%) of architecture transform results on CIFAR datasets. (B/M: billion/million)

\begin{tabular}{|c|c|c|c|c|c|c|c|}
\hline Method & Type & $\mathrm{C} 10+$ & $\mathrm{C} 100+$ & Params & Mults & Acts/image & Time/image \\
\hline \multicolumn{8}{|c|}{ ResNet-56 } \\
\hline Baseline & & 7.02 & 30.89 & $0.85 \mathrm{M}(1.0 \times)$ & $125.75 \mathrm{M}(1.0 \times)$ & $0.56 \mathrm{M}(1.0 \times)$ & $1.05 \mathrm{~ms}$ \\
\hline Recasting & Conv & 6.75 & 32.14 & $0.41 \mathrm{M}(2.1 \times)$ & $61.78 \mathrm{M}(2.0 \times)$ & $0.27 \mathrm{M}(2.0 \times)$ & $0.50 \mathrm{~ms}$ \\
\hline KD & Conv & 9.43 & 33.22 & $0.41 \mathrm{M}(2.1 \times)$ & $61.78 \mathrm{M}(2.0 \times)$ & $0.27 \mathrm{M}(2.0 \times)$ & $0.50 \mathrm{~ms}$ \\
\hline Backprop & Conv & 10.61 & 37.85 & $0.41 \mathrm{M}(2.1 \times)$ & $61.78 \mathrm{M}(2.0 \times)$ & $0.27 \mathrm{M}(2.0 \times)$ & $0.50 \mathrm{~ms}$ \\
\hline \multicolumn{8}{|c|}{ ResNet-83 } \\
\hline Baseline & & 6.34 & 28.13 & $0.83 \mathrm{M}(1.0 \times)$ & $125.09 \mathrm{M}(1.0 \times)$ & $1.69 \mathrm{M}(1.0 \times)$ & $1.51 \mathrm{~ms}$ \\
\hline Recasting & Conv & 6.90 & 31.04 & $0.41 \mathrm{M}(2.0 \times)$ & $61.78 \mathrm{M}(2.0 \times)$ & $0.27 \mathrm{M}(6.2 \times)$ & $0.95 \mathrm{~ms}$ \\
\hline KD & Conv & 8.95 & 32.75 & $0.41 \mathrm{M}(2.0 \times)$ & $61.78 \mathrm{M}(2.0 \times)$ & $0.27 \mathrm{M}(6.2 \times)$ & $0.95 \mathrm{~ms}$ \\
\hline Backprop & Conv & 9.77 & 37.14 & $0.41 \mathrm{M}(2.0 \times)$ & $61.78 \mathrm{M}(2.0 \times)$ & $0.27 \mathrm{M}(6.2 \times)$ & $0.95 \mathrm{~ms}$ \\
\hline \multicolumn{8}{|c|}{ WRN-28-10 } \\
\hline Baseline & & 4.06 & 19.54 & $36.45 \mathrm{M}(1.0 \times)$ & $5.24 \mathrm{~B}(1.0 \times)$ & $2.52 \mathrm{M}(1.0 \times)$ & $0.81 \mathrm{~ms}$ \\
\hline Recasting & Conv & 4.11 & 19.74 & $4.86 \mathrm{M}(7.5 \times)$ & $1.17 \mathrm{~B}(4.5 \times)$ & $0.90 \mathrm{M}(2.8 \times)$ & $0.41 \mathrm{~ms}$ \\
\hline KD & Conv & 4.40 & 19.94 & $4.86 \mathrm{M}(7.5 \times)$ & $1.17 \mathrm{~B}(4.5 \times)$ & $0.90 \mathrm{M}(2.8 \times)$ & $0.41 \mathrm{~ms}$ \\
\hline Backprop & Conv & 4.67 & 20.90 & $4.86 \mathrm{M}(7.5 \times)$ & $1.17 \mathrm{~B}(4.5 \times)$ & $0.90 \mathrm{M}(2.8 \times)$ & $0.41 \mathrm{~ms}$ \\
\hline \multicolumn{8}{|c|}{ DenseNet-100 } \\
\hline Baseline & & 5.11 & 23.62 & $0.74 \mathrm{M}(1.0 \times)$ & $0.29 \mathrm{~B}(1.0 \times)$ & $4.41 \mathrm{M}(1.0 \times)$ & $2.12 \mathrm{~ms}$ \\
\hline Recasting & Basic & 4.91 & 22.39 & $2.53 \mathrm{M}(0.3 \times)$ & $0.77 \mathrm{~B}(0.4 \times)$ & $0.89 \mathrm{M}(4.9 \times)$ & $0.27 \mathrm{~ms}$ \\
\hline $\mathrm{KD}$ & Basic & 4.71 & 22.71 & $2.53 \mathrm{M}(0.3 \times)$ & $0.77 \mathrm{~B}(0.4 \times)$ & $0.89 \mathrm{M}(4.9 \times)$ & $0.27 \mathrm{~ms}$ \\
\hline Backprop & Basic & 5.39 & 24.57 & $2.53 \mathrm{M}(0.3 \times)$ & $0.77 \mathrm{~B}(0.4 \times)$ & $0.89 \mathrm{M}(4.9 \times)$ & $0.27 \mathrm{~ms}$ \\
\hline Recasting & Conv & 6.82 & 25.60 & $0.87 \mathrm{M}(0.9 \times)$ & $0.19 \mathrm{~B}(1.5 \times)$ & $0.51 \mathrm{M}(8.6 \times)$ & $0.16 \mathrm{~ms}$ \\
\hline KD & Conv & 6.75 & 26.52 & $0.87 \mathrm{M}(0.9 \times)$ & $0.19 \mathrm{~B}(1.5 \times)$ & $0.51 \mathrm{M}(8.6 \times)$ & $0.16 \mathrm{~ms}$ \\
\hline Backprop & Conv & 8.11 & 30.05 & $0.87 \mathrm{M}(0.9 \times)$ & $0.19 \mathrm{~B}(1.5 \times)$ & $0.51 \mathrm{M}(8.6 \times)$ & $0.16 \mathrm{~ms}$ \\
\hline
\end{tabular}

Table 3: Error rates (\%) of compression results on CIFAR datasets. (B/M: billion/million)

\begin{tabular}{|c|c|c|c|c|c|c|c|}
\hline Method & Type & $\mathrm{C} 10+$ & C100+ & Params & Mults & Acts/image & Time/image \\
\hline \multicolumn{8}{|c|}{ VGG-16 } \\
\hline Baseline & & 6.85 & 28.80 & $14.71 \mathrm{M}(1.0 \times)$ & $313.20 \mathrm{M}(1.0 \times)$ & $0.31 \mathrm{M}(1.0 \times)$ & $0.37 \mathrm{~ms}$ \\
\hline Recasting & Conv & 8.31 & 31.56 & $2.36 \mathrm{M}(6.2 \times)$ & $50.63 \mathrm{M}(6.2 \times)$ & $0.13 \mathrm{M}(2.4 \times)$ & $0.31 \mathrm{~ms}$ \\
\hline KD & Conv & 9.24 & 33.14 & $2.36 \mathrm{M}(6.2 \times)$ & $50.63 \mathrm{M}(6.2 \times)$ & $0.13 \mathrm{M}(2.4 \times)$ & $0.31 \mathrm{~ms}$ \\
\hline Backprop & Conv & 8.71 & 35.13 & $2.36 \mathrm{M}(6.2 \times)$ & $50.63 \mathrm{M}(6.2 \times)$ & $0.13 \mathrm{M}(2.4 \times)$ & $0.31 \mathrm{~ms}$ \\
\hline \multicolumn{8}{|c|}{ WRN-28-10 } \\
\hline Baseline & & 4.06 & 19.54 & $36.45 \mathrm{M}(1.0 \times)$ & $5.24 \mathrm{~B}(1.0 \times)$ & $2.52 \mathrm{M}(1.0 \times)$ & $0.81 \mathrm{~ms}$ \\
\hline Recasting & Basic & 5.18 & 24.13 & $1.46 \mathrm{M}(24.9 \times)$ & $0.21 \mathrm{~B}(24.5 \times)$ & $0.52 \mathrm{M}(4.9 \times)$ & $0.56 \mathrm{~ms}$ \\
\hline KD & Basic & 5.48 & 25.28 & $1.46 \mathrm{M}(24.9 \times)$ & $0.21 \mathrm{~B}(24.5 \times)$ & $0.52 \mathrm{M}(4.9 \times)$ & $0.56 \mathrm{~ms}$ \\
\hline Backprop & Basic & 5.39 & 25.78 & $1.46 \mathrm{M}(24.9 \times)$ & $0.21 \mathrm{~B}(24.5 \times)$ & $0.52 \mathrm{M}(4.9 \times)$ & $0.56 \mathrm{~ms}$ \\
\hline
\end{tabular}

\section{ILSVRC2012}

For ILSVRC2012 dataset, we used the pre-trained ResNet50, DenseNet-121, and VGG-16 available from torchvision which is one of the PyTorch packages. These pretrained networks were used as the teacher networks. We recast the blocks of ResNet-50 into convolution blocks, and the blocks of DenseNet-121 into basic blocks. In addition, we recast only parts of these networks to obtain mixed-architecture networks. In Table 4, Recasting $(C)$ indicates that the student network only has convolution blocks, and Recasting $\left(C+R_{b t}\right)$ denotes that the student network has both convolution and bottleneck blocks. In the same way, Recasting $\left(R_{b s}\right)$ and Recasting $\left(R_{b s}+D\right)$ denotes that the student networks consist of only basic blocks and both basic blocks and dense blocks, respectively. $\mathrm{KD}\left(\mathrm{C}+\mathrm{R}_{b t}\right)$ and $\mathrm{KD}\left(\mathrm{R}_{b s}+\mathrm{D}\right)$ have the same network architecture as
Recasting $\left(\mathrm{C}+\mathrm{R}_{b t}\right)$ and Recasting $\left(\mathrm{R}_{b s}+\mathrm{D}\right)$ respectively, but those are trained with only KD method. For the VGG-16 compression, we used two criteria: higher parameter reduction $\left(\operatorname{Recasting}\left(C_{-} P\right)\right)$ and higher activation reduction $(R e-$ casting $\left.\left(C \_A\right)\right)$. In addition, we measured the actual inference time for all networks on an NVIDIA Titan X (Pascal) GPU, and batch sizes were set to 1 and 64 .

We measured the training time for Recasiting $\left(C+R_{b t}\right)$, $\mathrm{KD}\left(\mathrm{C}+\mathrm{R}_{b t}\right)$, Recasting $\left(\mathrm{R}_{b s}+\mathrm{D}\right)$ and $\mathrm{KD}\left(\mathrm{R}_{b s}+\mathrm{D}\right)$ to compare the training time and accuracy. Recasting $\left(\mathrm{C}+\mathrm{R}_{b t}\right)$ took 7.6 days, and $\mathrm{KD}\left(\mathrm{C}+\mathrm{R}_{b t}\right)$ took 6.3 days on a GPU. Compared to $\mathrm{KD}\left(\mathrm{C}+\mathrm{R}_{b t}\right)$, Recasting $\left(\mathrm{C}+\mathrm{R}_{b t}\right)$ took $20 \%$ longer, but achieved $2.00 \% \mathrm{p}$ and $0.59 \% \mathrm{p}$ improvement in top1 and top-5 accuracy, respectively. On the other hand, Recasting $\left(\mathrm{R}_{b s}+\mathrm{D}\right)$ took 3.9 days, while $\mathrm{KD}\left(\mathrm{R}_{b s}+\mathrm{D}\right)$ took 8.9 days with similar accuracy. Those results show that network recasting can achieve higher accuracy with slightly longer 
Table 4: Error rate (\%) of network recasting results on ILSVRC2012. (B/M: billion/million)

\begin{tabular}{|c|c|c|c|c|c|c|c|}
\hline Method & Top1 & Top5 & Params & Mults & Acts/image & Time/image & Time/bacth \\
\hline \multicolumn{8}{|c|}{ ResNet-50 } \\
\hline Baseline & 23.85 & 7.13 & $25.50 \mathrm{M}$ & $4.09 \mathrm{~B}$ & $11.57 \mathrm{M}$ & $6.16 \mathrm{~ms}$ & $107.17 \mathrm{~ms}$ \\
\hline Recasting(C) & 30.74 & 10.39 & $10.29 \mathrm{M}$ & $1.71 \mathrm{~B}$ & $2.53 \mathrm{M}$ & $2.12 \mathrm{~ms}$ & $37.21 \mathrm{~ms}$ \\
\hline $\operatorname{Recasting}\left(\mathbf{C}+\mathbf{R}_{b t}\right)$ & 25.00 & 7.71 & $21.72 \mathrm{M}$ & $2.40 \mathrm{~B}$ & $3.69 \mathrm{M}$ & $3.79 \mathrm{~ms}$ & $49.97 \mathrm{~ms}$ \\
\hline $\mathrm{KD}\left(\mathrm{C}+\mathrm{R}_{b t}\right)$ & 27.00 & 8.30 & $21.72 \mathrm{M}$ & $2.40 \mathrm{~B}$ & $3.69 \mathrm{M}$ & $3.79 \mathrm{~ms}$ & $49.97 \mathrm{~ms}$ \\
\hline \multicolumn{8}{|c|}{ DenseNet-121 } \\
\hline Baseline & 25.57 & 8.03 & $7.89 \mathrm{M}$ & $2.75 \mathrm{~B}$ & $16.52 \mathrm{M}$ & $12.73 \mathrm{~ms}$ & $111.31 \mathrm{~ms}$ \\
\hline Recasting $\left(\mathrm{R}_{b s}\right)$ & 26.42 & 8.25 & $32.23 \mathrm{M}$ & $8.15 \mathrm{~B}$ & $5.32 \mathrm{M}$ & $3.95 \mathrm{~ms}$ & $81.17 \mathrm{~ms}$ \\
\hline Recasting $\left(\mathrm{R}_{b s}+\mathrm{D}\right)$ & 24.87 & 7.59 & $10.42 \mathrm{M}$ & $5.72 \mathrm{~B}$ & $9.15 \mathrm{M}$ & $9.40 \mathrm{~ms}$ & $88.94 \mathrm{~ms}$ \\
\hline $\mathrm{KD}\left(\mathrm{R}_{b s}+\mathrm{D}\right)$ & 24.90 & 7.65 & $10.42 \mathrm{M}$ & $5.72 \mathrm{~B}$ & $9.15 \mathrm{M}$ & $9.40 \mathrm{~ms}$ & $88.94 \mathrm{~ms}$ \\
\hline \multicolumn{8}{|c|}{ VGG-16 } \\
\hline Baseline & 26.63 & 8.50 & $138.34 \mathrm{M}$ & $15.47 \mathrm{~B}$ & $15.09 \mathrm{M}$ & $6.17 \mathrm{~ms}$ & $200.47 \mathrm{~ms}$ \\
\hline Recasting(C_P) & 28.25 & 9.41 & $81.93 \mathrm{M}$ & $4.73 \mathrm{~B}$ & $8.27 \mathrm{M}$ & $3.45 \mathrm{~ms}$ & $116.45 \mathrm{~ms}$ \\
\hline Recasting(C_A) & 30.05 & 10.38 & $120.61 \mathrm{M}$ & $3.12 \mathrm{~B}$ & $3.30 \mathrm{M}$ & $3.61 \mathrm{~ms}$ & $63.52 \mathrm{~ms}$ \\
\hline
\end{tabular}

Table 5: Comparison of error rate (\%) with previous works on ILSVRC2012. (B/M: billion/million)

\begin{tabular}{|c|c|c|c|c|c|c|}
\hline Method & Top1 & Top5 & Params & Mults & Acts/batch & Actual speed-up \\
\hline \multicolumn{7}{|c|}{ ResNet-50 } \\
\hline $\operatorname{Rec}$ asting $\left(\mathrm{C}+\mathrm{R}_{b t}\right)$ & 25.00 & 7.71 & $21.72 \mathrm{M}$ & $2.40 \mathrm{~B}$ & $236.16 \mathrm{M}$ & $2.1 \times$ \\
\hline ThiNet-30 (Luo, Wu, and Lin 2017) & 31.58 & 11.7 & $8.66 \mathrm{M}$ & $1.10 \mathrm{~B}$ & - & $1.3 \times$ \\
\hline AutoPruner $(r=0.3)$ (Luo and $\mathrm{Wu} 2018)$ & 27.47 & 8.89 & - & $1.32 \mathrm{~B}$ & - & - \\
\hline \multicolumn{7}{|c|}{ VGG-16 } \\
\hline Recasting(C_A) & 30.05 & 10.38 & $120.61 \mathrm{M}$ & $3.12 \mathrm{~B}$ & $220.61 \mathrm{M}$ & $3.2 \times$ \\
\hline ThiNet-Conv (Luo, Wu, and Lin 2017) & 30.20 & 10.47 & $131.44 \mathrm{M}$ & $4.79 \mathrm{~B}$ & - & $2.5 \times$ \\
\hline RNP $(3 \times)($ Lin et al. 2017) & - & 12.42 & - & - & - & $2.3 \times$ \\
\hline Channel Pruning $(3 \times)(\mathrm{He}$, Zhang, and Sun 2017$)$ & - & 11.10 & - & - & - & $2.5 \times$ \\
\hline AutoPruner $(r=0.4)($ Luo and Wu 2018) & 31.57 & 11.57 & - & $4.09 \mathrm{~B}$ & - & - \\
\hline
\end{tabular}

training time for a deep network and shorter training time with similar accuracy for a shallow network.

As shown in Table 4, the network recasting significantly reduced the inference time in all experiments. Recasting $(\mathrm{C})$ and $\operatorname{Recasting}\left(\mathrm{R}_{b s}\right)$ achieved $2.9 \times$ and $3.2 \times$ inference time reduction for a single image compared with original ResNet-50 and DenseNet- 121, respectively. Moreover, mixed-architecture networks also achieved significant inference time reduction with smaller accuracy loss. For the batch processing, $\operatorname{Recasting}\left(\mathrm{C}+\mathrm{R}_{b t}\right)$ achieved $2.1 \times$ time reduction with $0.58 \%$ top-5 accuracy loss compared to Baseline, and Recasting $\left(\mathrm{R}_{b s}+\mathrm{D}\right)$ achieved $1.3 \times$ time reduction even with $0.44 \%$ higher top-5 accuracy. In particular, Recasting $\left(\mathrm{R}_{b s}+\mathrm{D}\right)$ achieved similar accuracy and inference time with $3.1 \times$ fewer parameters compared to $\operatorname{Recasting}\left(\mathrm{R}_{b s}\right)$. In VGG-16 compression, Recasting(C_P) and Recasting $\left(\mathrm{C} \_\mathrm{A}\right)$ achieved $1.7 \times$ parameter reduction and $4.6 \times$ activation reduction with $0.91 \%$ and $2.05 \%$ top-5 accuracy loss, respectively. Recasting(C_A) achieved $3.2 \times$ inference time reduction compared to the baseline.

We compared our results with several previous approaches (Luo, Wu, and Lin 2017; He, Zhang, and Sun 2017; Lin et al. 2017; Luo and Wu 2018). For the comparison, we used batch inference time because previous approaches have reported inference time only for the batch processing. Table 5 shows that the network recasting achieved much higher inference time reduction. In ResNet-50, Recasting $\left(\mathrm{C}+\mathrm{R}_{b t}\right)$ achieved lower error rate and much higher actual speedup compared with ThiNet (Luo, Wu, and Lin 2017). ThiNet only reduced filters and multiplications in $3 \times 3$ convolution of bottleneck blocks, so it cannot accelerate the inference effectively because activation load is still large. However, the network recasting can reduce the activation load effectively, so it achieved $2.1 \times$ actual speedup with smaller accuracy loss. Luo and $\mathrm{Wu}$ (2018) does not mention actual-speedup, but we can guess that our network recasting result is much faster than their AutoPruner result because they cannot remove the $1 \times 1$ convolution. For the VGG-16 compression, the network recasting also achieves much higher speedup with lower error rate compared to previous approaches. It also achieves higher parameter and multiplication reduction with similar accuracy compared to others.

\section{Conclusion}

In this paper, we proposed network recasting as a universal method for network architecture transformation. This 
method can accelerate network inference by transforming the network (teacher) to a more efficient one (student). We could recast residual and dense blocks into convolution and residual blocks, respectively, to achieve much higher actual speedup at small accuracy loss. By recasting blocks sequentially, the student network can be trained well even though there is no shortcut or dense connection. In addition, our method can recast arbitrary blocks, thereby producing a mixed-architecture network. The mixed-architecture networks produced as such achieved $2.1 \times$ inference time with $0.58 \%$ top-5 accuracy loss compared to original ResNet-50, and also achieved $1.3 \times$ inference time reduction with $0.44 \%$ higher top-5 accuracy on DenseNet-121 recasting. We also applied the network recasting for the purpose of compression and achieved higher compression ratio and speedup compared to previous approaches. Our method can be applied to various kinds of network architecture to transform it into various kinds of target network architecture.

\section{Acknowledgments}

This work was supported by Samsung Advanced Institute of Technology.

\section{References}

Ba, J., and Caruana, R. 2014. Do deep nets really need to be deep? In NIPS, 2654-2662.

Furlanello, T.; Lipton, Z. C.; Amazon, A.; Itti, L.; and Anandkumar, A. 2018. Born again neural networks. In ICML, 1607-1616.

Glorot, X., and Bengio, Y. 2010. Understanding the difficulty of training deep feedforward neural networks. In AISTATS, 249-256.

Goodfellow, I.; Bengio, Y.; Courville, A.; and Bengio, Y. 2016. Deep learning, volume 1. MIT press Cambridge.

Guo, Y.; Yao, A.; and Chen, Y. 2016. Dynamic network surgery for efficient DNNs. In NIPS, 1379-1387.

Han, S.; Pool, J.; Tran, J.; and Dally, W. 2015. Learning both weights and connections for efficient neural network. In NIPS, 1135-1143.

He, K.; Zhang, X.; Ren, S.; and Sun, J. 2016. Deep residual learning for image recognition. In $C V P R, 770-778$.

He, Y.; Zhang, X.; and Sun, J. 2017. Channel pruning for accelerating very deep neural networks. In ICCV, 13891397.

Hinton, G.; Vinyals, O.; and Dean, J. 2014. Distilling the knowledge in a neural network. In NIPS Deep Learning and Representation Learning Workshop.

Hornik, K. 1991. Approximation capabilities of multilayer feedforward networks. Neural networks 4(2):251-257.

Hu, H.; Peng, R.; Tai, Y.-W.; and Tang, C.-K. 2016. Network trimming: A data-driven neuron pruning approach towards efficient deep architectures. arXiv preprint arXiv: 1607.03250.

Hu, J.; Shen, L.; and Sun, G. 2018. Squeeze-and-excitation networks. In $C V P R, 7132-7141$.
Huang, G.; Liu, Z.; Weinberger, K. Q.; and van der Maaten, L. 2017. Densely connected convolutional networks. In CVPR, 4700-4708.

Ioffe, S., and Szegedy, C. 2015. Batch normalization: Accelerating deep network training by reducing internal covariate shift. In ICML, 448-456.

Kingma, D. P., and Ba, J. 2015. Adam: A method for stochastic optimization. In ICLR.

Larsson, G.; Maire, M.; and Shakhnarovich, G. 2017. Fractalnet: Ultra-deep neural networks without residuals. In ICLR.

Li, H.; Kadav, A.; Durdanovic, I.; Samet, H.; and Graf, H. P. 2016. Pruning filters for efficient convnets. In ICLR.

Lin, J.; Rao, Y.; Lu, J.; and Zhou, J. 2017. Runtime neural pruning. In NIPS, 2181-2191.

Lin, M.; Chen, Q.; and Yan, S. 2014. Network in network. In ICLR.

Liu, B.; Wang, M.; Foroosh, H.; Tappen, M.; and Pensky, M. 2015. Sparse convolutional neural networks. In $C V P R$, 806-814.

Liu, Z.; Li, J.; Shen, Z.; Huang, G.; Yan, S.; and Zhang, C. 2017. Learning efficient convolutional networks through network slimming. In ICCV, 2755-2763.

Luo, J.-H., and Wu, J. 2018. AutoPruner: An end-to-end trainable filter pruning method for efficient deep model inference. arXiv preprint arXiv: 1805.08941.

Luo, P.; Zhu, Z.; Liu, Z.; Wang, X.; and Tang, X. 2016. Face model compression by distilling knowledge from neurons. In $A A A I, 3560-3566$.

Luo, J.-H.; Wu, J.; and Lin, W. 2017. ThiNet: A filter level pruning method for deep neural network compression. In ICC V, 5058-5066.

Romero, A.; Ballas, N.; Kahou, S. E.; Chassang, A.; Gatta, C.; and Bengio, Y. 2015. FitNets: Hints for thin deep nets. In $I C L R$.

Simonyan, K., and Zisserman, A. 2015. Very deep convolutional networks for large-scale image recognition. In ICLR.

Sutskever, I.; Martens, J.; Dahl, G.; and Hinton, G. 2013. On the importance of initialization and momentum in deep learning. In ICML, 1139-1147.

Szegedy, C.; Liu, W.; Jia, Y.; Sermanet, P.; Reed, S.; Anguelov, D.; Erhan, D.; Vanhoucke, V.; and Rabinovich, A. 2015. Going deeper with convolutions. In CVPR, 1-9.

Yim, J.; Joo, D.; Bae, J.; and Kim, J. 2017. A gift from knowledge distillation: Fast optimization, network minimization and transfer learning. In CVPR, 4133-4141.

Zagoruyko, S., and Komodakis, N. 2016. Wide residual networks. In $B M V C, 87.1-87.12$.

Zagoruyko, S., and Komodakis, N. 2017. Paying more attention to attention: Improving the performance of convolutional neural networks via attention transfer. In ICLR. 